\title{
Hybrid Heterogeneous Energy Supply Networks
}

\author{
Farinaz Koushanfar and Azalia Mirhoseini \\ Department of Electrical \& Computer Engineering, Rice University, Houston, TX \\ \{farinaz,azalia\}@ece.rice.edu
}

\begin{abstract}
Efficient energy supply, storage, and distribution are key technical challenges for design and operation of electronic systems. In particular, energy supply is the most scarce resource and constraint for mobile embedded systems where the supply lifetime, cost, weight, size, and portability are major concerns. With the advent of newer electrical energy supply and storage technologies, energy supplies with differing energy/power storage densities, cost, size, and recycling abilities are becoming available. Efficient system design requires an exact matching between the fluctuating load demands and the underlying energy resources. Combining the energy supplies in a hierarchical way creates a unique opportunity for efficient matching and variable load serving. Such a heterogeneous hybrid network of energy supply components could address a variety of power needs and serve a much broader range of system loads with a high efficiency. This paper presents a Hierarchial Architecture of Heterogenous Electrical Energy Supplies (HierArcHEES) that can be tuned to different load demands.
\end{abstract}

\section{INTRODUCTION}

A mobile system's life duration and functionality is bounded by its constrained energy source. Most of the contemporary portable and mobile systems, only contain one type of energy supply in form of an electrochemical battery or simply a battery. While scaling of the electronic and semiconductor components has resulted in an exponential growth in computational capacity over the past few decades, the battery technology and its energy capacity (i.e., total energy stored in the device) have only linearly progressed with a slow growth rate. The conventional energy supply systems also have a number of other limitations including the slow charge cycles, limited lifetime, and low power densities.

Recent technological advances are promising new forms of energy supply and storage with characteristics that are vastly differing from the conventional batteries. The idea of a hierarchical architecture of heterogeneous energy supply system along with a high level architecture was first proposed in [1]. The goal is to overcome the limitations of a single energy source since it is unlikely that a single energy supply would simultaneously optimize all the criteria imposed by the load demand. The idea was inspired by studying the hierarchical processor memory system where multiple storage options, including but not limited to FLASH memory, SRAM, caches, and external disks are often simultaneously available. The performance metrics for determining the best storage option includes speed of access, size, power, density, and memory permanency. Depending on the task at hand, an interleaved combination of the memory subsystems could be used. The analogy between the processor hierarchical memory architecture (serving differing storage requirements) and the proposed energy supply network was used to motivate the need for multiple energy sources [1]. A later independently proposed system called hybrid electrical energy storage (HEES) also suggested a block-level system realization for a hybrid network of energy supply options [2]. Recently a hybrid battery-supercapacitor power-supply system has been proposed in which the hybrid source's charge/discharge policy is made based upon the load profile and supply's state. The work includes a novel algorithm to maximize the supply system's lifetime [3]. A number of limited earlier studies have already shown the benefits of a hybrid supercapacitor and solar energy generation system in optimizing system's efficiency and dynamic response time, in particular for transient loads with a low duty cycle and also for pulsed loads in sensor networks [4], [5], [6].

In this paper, we elaborate on the hierarchical energy supply network design suggested in [1] and provide more details for its system macro-architecture. We use the term HierArcHEES to refer to our Hierarchial Architecture of Heterogenous Electrical Energy Supplies. The HierArcHEES system includes different energy supply and storage units along with multiple control units, where the system allows a flexible matching of the appropriate energy supply/storage option to the workload demand.

We start by introducing a number of performance metrics that are of high importance while designing an energy supply network. Next, we discuss the present electronic energy supply and storage technologies and provide a brief discussion on the similarities and differences among them. The HierArcHEES system exploits the performance metrics to include a hybrid combination of the energy supply/storage options within its system architecture while exploiting the best properties of each type of energy supply. We discuss the parameters of the HierArcHEES system, the challenges associated with designing and realizing this system and ongoing research problems.

The remainder of the paper is organized as follows. In Section II, we present the criteria for selecting energy supply and storage devices. Section III provides an overview of the electrical energy storage technologies. The macro-architecture details of the HierArcHEES system is demonstrated in Section IV. We discuss the challenges in design and realization of HierArcHEES, and opportunities for newer developments in Section V. We conclude in Section VI.

\section{PERformance Metrics}

In this section, we outline a number of important metrics that are commonly used for evaluating the efficiency of an energy supply/storage system:

- Energy and power density: Energy density quantifies the amount of stored energy in a unit of volume (or weight) 
of an energy supply/storage device. Power density often characterizes the average value of rate of energy change (or mean available power) for a unit of volume (weight) of the device.

- Charge time and discharge delivery rate: The Charge time is the time duration needed to transfer charges to an energy storage device such that it reaches the full energy capacity. The output power rate, or discharge delivery rate determines the time needed to extract one unit of the stored energy. The discharge time, that is the maximumpower discharge duration that is defined as the ratio of the overall energy capacity to the maximum power.

- Leakage (self-discharge) rate: This measure quantifies the rate of charge leakage when not used. The selfdischarge rate of a device is important since it determines how long an energy storage device can stay idle. The leakage, which varies a lot across the spectrum of available energy storage options, is a deciding factor for selecting the best energy source to supply the load.

- Efficiency: This is the ratio between the utilized energy and the stored energy. The cycle efficiency may also be defined as the product of charging efficiency and discharging efficiency. In a highly efficient system, the power transfer system must have small losses due to energy conversion/transfer or self-discharge. The per cycle efficiency of a device is often a complex relationship varying with the operation point such as ambient temperature and notably with the state of charge. Also, the method of charging or discharging can affect efficiency.

- Durability (cycle capacity) and aging: This metric quantifies the number of times an energy storage unit can release the energy level it was designed for (after each recharge). The cycle capacity may be expressed as the maximum number of cycles, where a cycle corresponds to once charging and once discharging the unit. The durability of an energy supply/storage unit often deteriorates with aging of the device. Aging is related to wear by usage (irreversible chemical and physical modifications that occur during operation), and it is also related to state of charge and operation point. Modeling the aging process is often complex and not well-defined.

- Autonomy: This metric defines the maximum amount of time the system can continuously release energy once it is charged to its full energy capacity.

- Cost, volume, and weight: The cost is one of the most important metrics for selecting energy sources while devising a system. The most commonly used quantity for the expense metric is cost per unit of energy stored, or the cost per unit of mean output power. The cost per unit of energy supply or storage device may also be characterized versus the unit of pertinent device's volume or its weight. While designing a hybrid energy supply system, the overall cost of the system may be a design constraint to keep the overall electronic system economically viable.

- Reliability and safety: While the reliability and safety are more subjective than the metrics defined so far, they constitute an important dimension in selecting the appropriate energy source. For example, in certain scenarios, it may be possible to use energy scavenging and then convert/store the resulting energy in a supercapacitor. However, such an intermittent energy scavenging option may be unreliable for critical applications that need a constant source. Safety dictates another set of subjective concerns. For example, in high temperatures, fuel cells that might explode should be excluded even if they satisfy the other design metrics.

- Adaptability: In the context of a specific application load, the adaptability of the energy system is the ability of the energy source to match the application requirements.

- Other characteristics: While the metrics defined above provide a good set of evaluation criteria for a generic energy storage system, other evaluation methods, especially those specific to an energy supply or storage technology may be used in real application scenarios. Another set of specific metrics are related to the application requirements. Such metrics are less general and outside the scope of the current paper.

\section{STATE-OF-THE-ART AND EMERGING TECHNOLOGIES}

\section{A. Electrochemical batteries}

A battery consists of electrochemical cells that convert stored chemical energy into electrical energy. Examples of commercial off-the-shelf electrochemical batteries include Lead-acid batteries, Li-ion batteries, NiMH batteries, and Metal-air batteries [2].

Lead acid batteries are currently used in most automobiles for starting, lighting, and ignition applications. They have been used in electric vehicles including General Motors' EV-I. Lead acid batteries are very efficient and inexpensive, however, they have low energy densities, $[10,50] W h / K g$, power densities in the range of $[1,500] \mathrm{W} / \mathrm{kg}$, and poor lifetime.

$\mathrm{NiMH}$ batteries can provide high power densities up to $1300 \mathrm{~W} / \mathrm{kg}$ and energy densities up to $110 \mathrm{Wh} / \mathrm{kg}$. NiMH batteries have reasonable prices and have enabled commercial HEVs. They have exceptional high temperature capability and long-life under high depth of discharge. Their main disadvantage is that they suffer from memory effect.

Lithium-ion batteries are used in most contemporary consumer electronics. High efficiency, long life-cycle and low self-discharge rate are their advantages. Recent advances have enabled production of Lithium-ion batteries with extremely high power densities such as Sony's $1800 \mathrm{~W} / \mathrm{kg}$ batteries and energy densities of up to $250 \mathrm{Wh} / \mathrm{kg}$. The main disadvantage of those batteries is their relatively expensive prices.

\section{B. Capacitors}

A capacitor is a passive electronic component consisting of a pair of conductors separated by a dielectric. It can store electric energy when disconnected from its charging circuit, so it can be used like a temporary battery. They are commonly used in electronic devices to maintain power supply DC level while batteries are being changed. Capacitors power densities and charge/discharge rates are higher than that of batteries and supercapacitors but they have lower energy densities. 


\section{Supercapacitors}

A supercapacitor based on electrochemical double layer capacitance is another electrical energy storage device that has a much higher energy density compared to conventional capacitors. Supercapacitors can store a large amount of charge and deliver it at much higher power rates than batteries. Their benefits other than high power and energy density are high cycle efficiency, long cycle life, low maintenance and wide thermal operating range. However supercapacitors' energy densities are still lower than batteries or fuel cells. The other shortcoming is the low voltage they can provide comparing to the conventional capacitors. Size of the supercapacitors is another concern, however the recent progress in graphenebased materials and nano-technology has raised hopes for using them as power supplies [7].

\section{Fuel cells}

Fuel cell is an electrochemical energy device that converts a fuel source into electricity. The main advantages of fuel cells are their considerably high energy density, environmentfriendly system and low maintenance. A number of currently under development fuel cells such as the polymer electrolyte membrane fuel cells (PEMFC)s can be used for stationary, portable, and mobile applications. Some of their disadvantages are cost, low power density, and low cycle efficiency in comparison with batteries and supercapacitors. Currently they can provide cycle efficiencies of up to 65 percent.

Table 1 shows a numerical comparison between the present energy performance metrics of different power supplies. It is observable that no single unit outperforms others in all the metrics and the advantage of a hybrid system is evident.

TABLE I

A COMPARISON OF DIFFERENT POWER SUPPLY PERFORMANCE METRICS.

\begin{tabular}{|c|c|c|c|c|}
\hline Property & Supercap & Capacitor & Battery & $\mu$ FuelCell \\
\hline Life & $>30 k h \mathrm{r}$ & $>100 k$ cycles & {$[1.5-10] \mathrm{khr}$} & {$[.15-1.5] \mathrm{k} \mathrm{cycles}$} \\
\hline Cycle & {$\left[10^{-3}, 1\right] \mathrm{s}$} & {$\left[10^{-9}, 1\right] \mathrm{ms}$} & {$[1,10] \mathrm{hr}$} & {$[10,300] \mathrm{hr}$} \\
\hline$W e i g h t$ & $1-2 \mathrm{~g}$ & $1 \mathrm{~g}-10 \mathrm{~kg}$ & $1 \mathrm{~g}-10 \mathrm{~kg}$ & $25 \mathrm{~g}-5 \mathrm{~kg}$ \\
\hline$\$ / \mathrm{kWhr}$ & {$[20-50] \mathrm{k}$} & - & {$[10,2500]$} & {$[3.5,10] \mathrm{k}$} \\
\hline$P \rho, \mathrm{kW} / \mathrm{kg}$ & {$[10,100]$} & {$\left[.25,10^{4}\right]$} & {$[.005,1.8]$} & {$[.001,1]$} \\
\hline$E \rho, W h r / K g$ & {$[1,28]$} & {$[.01, .05]$} & {$[8,600]$} & {$[.3,3] \mathrm{k}$} \\
\hline
\end{tabular}

\section{HIERARCHEES SYSTEM}

In this section, we introduce HierArcHEES, a modular architecture for Hybrid energy source system. Our new hybrid architecture aims at combining a heterogeneous set for energy supply elements. The rationale behind selecting a heterogeneous set is that no one element can provide all the desired performance criteria. Combining the energy resources in a hierarchical way is a viable approach to simultaneously integrate the best properties of all available energy supply and storage elements within one framework.

A three-tier hierarchy of energy supply and storage elements is exploited in the HierArcHEES system:

1) External energy supplies: The first tier includes the outside sources that are not within the closed electronic

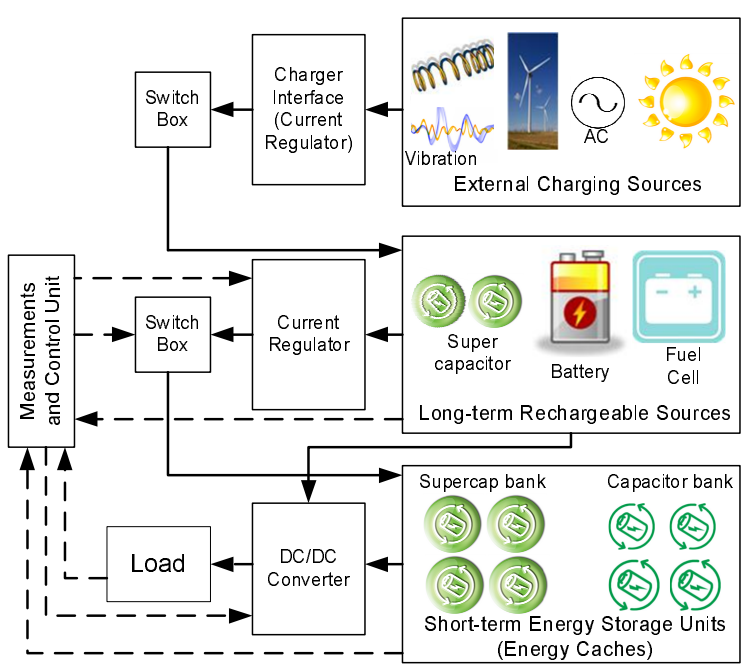

Fig. 1. HierArcHEES system.

systems, but they inject energy to the system. The external sources include (but are not limited to) the grid, AC sources, or non-rechargeable chemical batteries. Other external sources such as solar energy harvesting and vibration energy scavenging can also convert the energy to electrical energy that will be saved in long-term rechargeable supply elements.

2) Long-term rechargeable energy supply and storage elements: At the second tier of hierarchy are elements that can be recharged by the external sources. A recharged source can be disconnected to allow system's closeness and portability (i.e., disconnection from the external sources) for a long time duration. The length of time is a subjective measure that is a function of technology and application. For example, for the presently available embedded mobile systems, a recharged battery would last in an order of multiple hours, and sometimes even multiple days depending on the application.

3) Short-term recycling energy storage elements: At the third tier of hierarchy are the energy recycling elements that are typically more than an order of magnitude faster charged (typically at the expense of smaller energy density and less autonomy) compared with the conventional long-term rechargeable elements. They can be used for intermediate storage and recycling of charges drawn from the long-term energy elements, while they have a longer life cycle, higher power density, better charge delivery, and better efficiency.

Figure 1 demonstrates a flow diagram for the HierArcHEES system. We note here that each element in the tier may be a bank of elements that can be reconfigured to provide a desired current/voltage signal. In addition to the three-tier energy supply and storage hierarchy, there are a number of control and leveling components needed to ensure the correct functionality of the hybrid energy supply system. Perhaps the most important component in the HierArcHEES system is the control and measurement units(s). Depending on the application at hand, the control and measurement unit may be 
centralized or distributed. One important task of the control and measurement unit, is supervising the charge transfer. As shown on the figure, connecting the external energy sources to the second tier components and connecting the secondtier components to the third tier require charging interfaces controlled by the management. This is because charge transfer to and from each of the heterogeneous energy elements needs specific voltage and current inversion to ensure the cycle life efficiency of the energy source and safety.

The key task of the control and measurement unit(s) is to decide how to serve the load considering the heterogeneous resources in the HierArcHEES system. The control is based on the measurements of the load's shape and its demands, and the performance metrics of the elements of power supply system in addition to the present state of the system (e.g., state-ofcharge of energy supply elements), and supply reconfiguration overhead. While setting the load is the main task, another important role of the energy measurement and control system is to monitor and supervise charge transfer among the energy supply and storage elements in the three levels of our hierarchy. The control management of transfer charges is shown by switches on the figure. Since voltage and current adjustment are sensitive tasks and often require feedback to mitigate the impact of instantaneous fluctuations, PIDs and other feedback structures are typically used.

\section{Challenges AND OPPORTUNities}

The architecture demonstrated in the previous section enables a number of new applications. Simultaneously, it requires design and operation of a rather complex system. In addition to finding the right applications that can benefit from this hybrid paradigm, there are a set of challenges associated with designing and operating the HierArcHEES system in practical scenarios that also provide opportunities for further research. Orthogonal to research in energy supply/storage technology, the important directions that should be investigated include:

- Modeling the energy sources: An important step in selecting and operating the energy supply and storage elements is knowing the performance metrics. Extensive measurements in different conditions are needed for source characterization and performance evaluation. Modeling of the sources with fidelity to the physical and chemical phenomena is a challenging task that typically requires knowledge from mathematics, physics, chemistry, and statistics.

- Load characterization: The load's shape has a detrimental impact on the energy drawn from the supply. It simultaneously provides us with opportunities to adjust the sources to the demand. Profiling the load or correctly predicting its future behavior in real time and during the system operation is a challenge. There is a need to develop methods for fast load estimation, so the resources can be correctly assigned.

- Charging and power transfer/conversion: Finding the best time to charge the rechargeable sources is not always deterministic. It can be a function of the load, availability of the external sources, and the state of the upper tier components. Methods for mitigating the overhead of charge transfer and for lowering the leakage are mandatory for achieving an efficient supply system.

- Control and management: We have already emphasized on the importance of the control and management unit(s). The tasks for this important unit includes coordinating the charging/discharging or disconnection of the energy supply elements, developing intelligent algorithms for instantaneously matching the load to the pertinent resources, management of charge storage and transfer between the different tiers, and voltage/current conversion. Improvements at the circuit level, technology, control algorithms, real-time management, and modeling are needed to address the challenges.

\section{CONCLUSION}

A hybrid energy supply is a system comprised of heterogeneous energy supply elements with differing performance characteristics. We provided a taxonomy of the important characteristics of the energy supply elements including energy density, power density, durability, self-discharge, autonomy, efficiency, charge transfer rate, rate capacity effect, adaptability, and cost effectiveness. We also provided a summary of the commonly available technologies for energy storage elements including electrochemical batteries, capacitors, supercapacitors, and fuel cells and provided a brief comparison between them. A three-tier hierarchical system, called HierArcHEES was introduced for better matching of the underlying energy resources to the application demands. This paper outlined a number of challenges and opportunities for further research and development on this topic. Given the continuous technological advances, the success of hierarchical architecture in processor memory management for many years, and the surge of interest on this topic, the proposed hybrid hierarchical energy supply system with heterogenous elements could address a spectrum of energy demands with varying characteristics and serve a much broader load with a highly improved efficiency.

\section{ACKNOWLEDGEMENT}

This research is in parts supported by the Office of Naval Research (ONR) YIP award (grant No. R16480) and National Scient Foundation CAREER award (grant No. R3A530).

\section{REFERENCES}

[1] F. Koushanfar, "Hierarchical hybrid power supply networks," in $D A C$, 2010, pp. 629-630

[2] M. Pedram, N. Chang, Y. Kim, and Y. Wang, "Hybrid electrical energy storage systems," in ISLPED, 2010, pp. 363-368.

[3] A. Mirhoseini and F. Koushanfar, "Hypoenergy: Hybrid supercapacitorbattery power-supply optimization for energy efficiency," in DATE, 2011.

[4] D. Brunelli, C. Moser, L. Thiele, and L. Benini, "Design of a solarharvesting circuit for batteryless embedded systems," TCAS I: Regular Papers, vol. 56, no. 11, pp. 2519-2528, 2009.

[5] G. Merrett, A. Weddell, A. Lewis, N. Harris, B. Al-Hashimi, and N. White, "An empirical energy model for supercapacitor powered wireless sensor nodes," in ICCCN, 2008, pp. 1-6.

[6] T. Zhu, Z. Zhong, Y. Gu, T. He, and Z. Zhang, "Leakage-aware energy synchronization for wireless sensor networks," in MobiSys, 2009, pp. 319332.

[7] Y. Wang, Z. Shi, Y. Huang, Y.Ma, C. Wang, M. Chen, and Y. Chen, "Supercapacitor devices based on graphene materials," American Chemical Society, J. Phys. Chem, pp. 13 103-13 107, 2009. 